\title{
Static and Dynamic Parameters in Patients With Degenerative Flat Back and Change After Corrective Fusion Surgery
}

\author{
Jung Hwan Lee, MD, $\mathrm{PhD}^{1}$, Sang-Ho Lee, $\mathrm{MD}, \mathrm{PhD}^{2}$ \\ Departments of ${ }^{1}$ Physical Medicine and Rehabilitation, ${ }^{2}$ Neurosurgery, Wooridul Spine Hospital, Seoul, Korea
}

\begin{abstract}
Objective To evaluate characteristics of static and dynamic parameters in patients with degenerative flat back (DFB) and to compare degree of their improvement between successful and unsuccessful surgical outcome groups Methods Forty-seven patients with DFB were included who took whole spine X-ray and three-dimensional motion analysis before and 6 months after corrective surgery. Forty-four subjects were selected as a control group. As static parameters, thoracic kyphosis (TK), thoracolumbar junction (TLJ), lumbar lordosis (LL), pelvic incidence (PI), sacral slope (SS), and pelvic tilt (PT) were measured. As dynamic parameters, maximal and minimal angle of pelvic tilt, lower limb joints, and thoracic and lumbar vertebrae column (dynamic TK and LL) in sagittal plane were obtained.

Results The DFB group showed smaller TK and larger LL, pelvic posterior tilt, hip flexion, knee flexion, and ankle dorsiflexion than the control group. Most of these parameters were significantly corrected by fusion surgery. Dynamic spinal parameters correlated with static spinal parameters. The successful group obtained significant improvement in maximal and minimal dynamic LL than the unsuccessful group.

Conclusion The DFB group showed characteristic lower limb and spinal angles in dynamic and static parameters. Correlation between static and dynamic parameters was found in spinal segment. Dynamic LL was good predictor of successful surgical outcomes.
\end{abstract}

Keywords Degeneration, Motion, Spinal fusion, Treatment outcome

\section{INTRODUCTION}

Degenerative flat back (DFB) refers to a spinal sagittal imbalance due to decreased lumbar lordosis caused by degenerative changes and various disabilities in daily living associated with stooped posture [1,2]. Although DFB is very rare in Western countries, it is a common spinal deformity in Asian countries. The cause of this ethnical

Received September 30, 2015; Accepted January 15, 2016

Corresponding author: Jung Hwan Lee

Department of Physical Medicine and Rehabilitation, Wooridul Spine Hospital, 445, Hakdong-ro, Gangnam-gu, Seoul 06068, Korea. Tel: +82-2-5138151, Fax: +82-2-513-8146, E-mail: j986802@hanmail.net

ORCID: Jung Hwan Lee (http://orcid.org/0000-0003-2680-6953); Sang-Ho Lee (http://orcid.org/0000-0002-8526-0260).

@ This is an open-access article distributed under the terms of the Creative Commons Attribution Non-Commercial License (http://creativecommons.org/ licenses/by-nc/4.0) which permits unrestricted noncommercial use, distribution, and reproduction in any medium, provided the original work is properly cited. Copyright $\odot 2016$ by Korean Academy of Rehabilitation Medicine 
difference is explained by different lifestyles, such as sitting on floor or working while squatting. Most patients reveal four cardinal and characteristic clinical symptoms: stooping while walking, inability to lift heavy objects in front, difficulty in climbing slopes, and the need to support oneself with the elbow, resulting in formation of a hard corn on the extensor surface of the elbow [3]. Although the exact pathophysiology of DFB has not yet been confirmed, extensive degeneration and weakness of lumbar extensor muscles are thought to be responsible for that condition in most patients $[2,4]$.

Because radiographic examinations reveal characteristic sagittal deviation of spinopelvic alignment, diagnosis of DFB is usually made on the basis of clinical and radiographic findings [3]. Decreased lumbar lordosis causes anterior displacement of the center of gravity, which leads to spinopelvic angular changes and further affects standing posture and gait characteristics $[5,6]$. The studies regarding spinopelvic imbalance in the patients with DFB have focused mainly on static parameters measured by simple radiography $[3,7-9]$. However, radiography has the limitation that it can only reveal the static posture, and cannot evaluate the dynamic status, such as ambulation.

However, daily activities or functional aspects of patients are more related to dynamic status of the spinopelvic segment than static posture measured by radiography. Treatment outcomes or patient satisfaction is also influenced by dynamical parameters rather than static parameters. Thus, it is assumed that assessment of dynamic parameters of DFB can provide clinically useful data about patients' functional status and treatment outcomes. Three-dimensional (3D) motion analysis has been a useful tool to analyze biomechanical parameters in various conditions including spinal problems [10-13]. It is hypothesized that $3 \mathrm{D}$ motion analysis can provide specific characteristics of spinopelvic and lower limb joint motion in patients with DFB, and also reveal which improvement would occur following surgeries in a quantitative and objective way. Previous studies investigated dynamic parameters in the patients with DFB but they did not assess dynamic parameters of spinal segments and did not perform motion analysis test after corrective surgeries, so they could not evaluate the improvement acquired by surgical treatments $[5,14]$.

The purposes of this study were (1) to evaluate the static spinopelvic alignment on a plain radiography and the dynamic spinal status and lower limb kinematics by 3D motion analysis of the patients with DFB compared with normal control; (2) to evaluate the improvement of static and dynamic parameters after corrective fusion surgeries in comparison with those of pretreatment; (3) to assess the correlation between the changes of static and dynamic parameter improvements after corrective surgeries; and (4) to compare the amount of the improvement of static and dynamic parameters between the successful and unsuccessful surgical outcome groups.

\section{MATERIALS AND METHODS}

\section{Patients}

This study was approved by the Institutional Review Board of Wooridul Spine Hospital. This retrospective study was based on medical chart reviews of the patients who had undergone corrective fusion surgeries for the treatment of DFB from 2010 to 2012. The surgeries were decided mainly on clinical and radiological basis. They were indicated in the patients who suffered from severe pain and difficulty in ambulation and daily activities with radiological sagittal imbalance. The 68 patients who received a whole spine X-ray and 3D motion analysis before and 6 months after corrective surgeries were chosen. Of those, 10 patients with prominent knee or hip pain and 5 with lower limb weakness were excluded because their conditions might affect gait analysis results. In addition, 6 patients who had undergone previous lumbar surgery before corrective surgery were also excluded because previous surgery might change spinopelvic parameters. Finally, 47 patients were included in this study and were classified as the DFB group.

The DFB group was divided into successful and unsuccessful surgical outcomes groups according to the modified MacNab criteria, the rating scale of treatment outcomes in terms of patient's subjective satisfaction [15]. The explanations of each grade was as follows: excellent (no pain, no restriction of mobility, return to normal work and level of activity), good (occasional nonradicular pain, relief of presenting symptoms, able to return to modified work), fair (some improved functional capacity, still handicapped and/or unemployed), and poor (continued objective symptoms of root involvement, additional operative intervention needed at index level 
irrespective of length of postoperative follow-up).

A successful outcome was defined as excellent or good based on the modified MacNab criteria [16]. Forty-four subjects (38 women and 6 men) without spinal and lower extremity pathology were included as a control group and took a whole spine $\mathrm{X}$-ray and a $3 \mathrm{D}$ motion analysis (control group) in the same way with the DFB group.

\section{Radiological evaluation (static parameters)}

Spinal sagittal parameters of thoracic kyphosis (TK), thoracolumbar junction (TLJ), and lumbar lordosis (LL) were measured in a whole spine lateral view. TK was measured from the superior end plate of T5 to the inferior end plate of T12. TLJ was measured from the superior end plate of T10 to the inferior end plate of L2. LL was measured from the inferior end plate of $\mathrm{T} 12$ to the supe- rior end plate of S1 by the Cobb method (Fig. 1A). Pelvic parameters of pelvic incidence (PI), sacral slope (SS), and pelvic tilt (PT) were measured. SS represented the angle between the superior end plate of $S 1$ and a horizontal line. PT was defined as the angle between a vertical line originating at the center of the bicoxofemoral axis and a line drawn between the same point and the middle of the superior end plate of S1. PI was defined as the angle between the line perpendicular to the sacral plate and the line connecting the midpoint of the sacral plate to the bicoxofemoral axis [3,17] (Fig. 1B). All parameters were measured at standing position because sagittal angular deviation of DFB was correctly obtained in weight-bearing position.
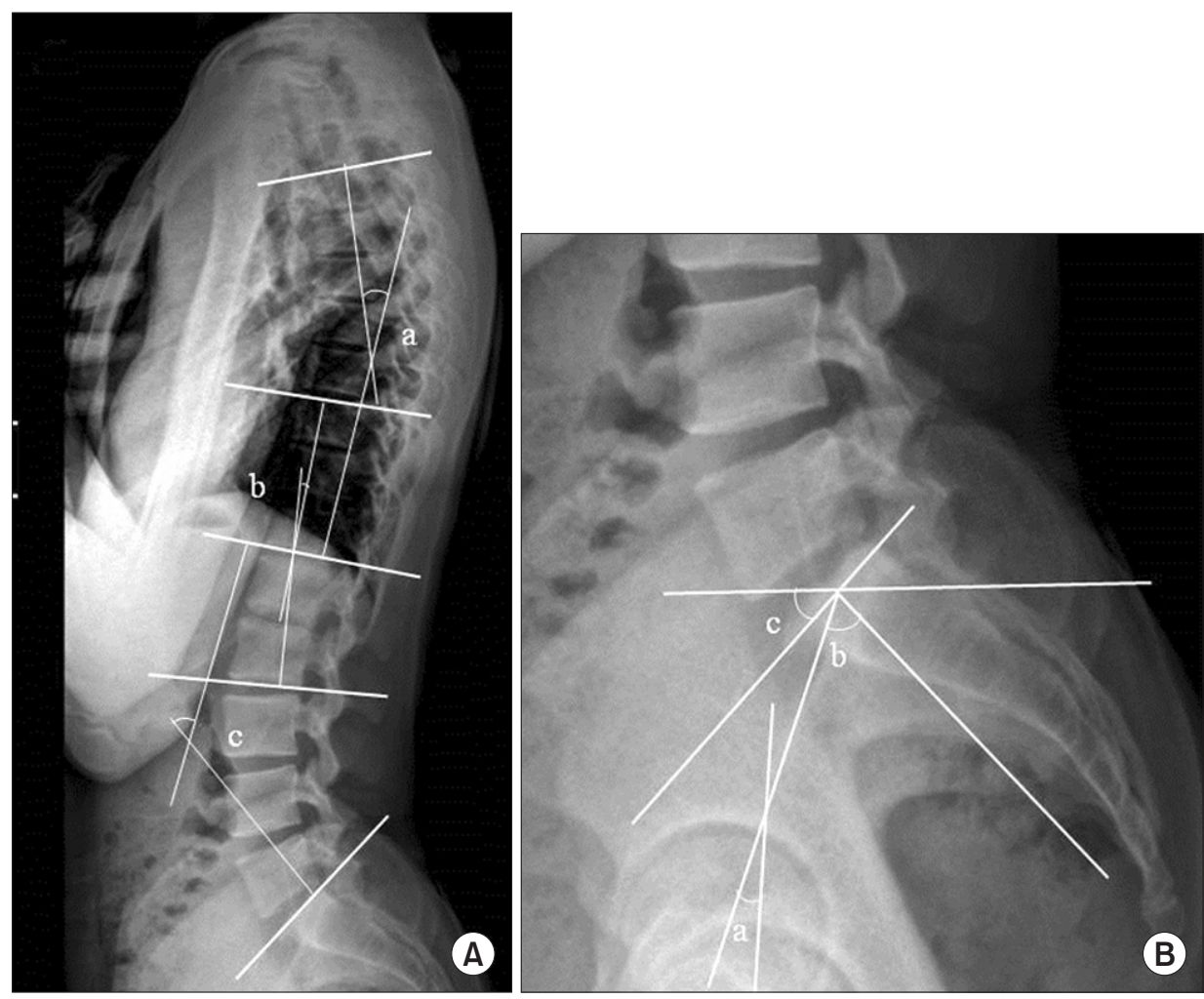

Fig. 1. Spinal and pelvic parameters. (A) Spinal parameters including thoracic kyphosis (TK), thoracolumbar junction (TLJ), and lumbar lordosis (LL). (a) TK was measured from the T5 superior end plate to T12 inferior end plate. (b) TLJ was measured from the T10 superior end plate to L2 inferior end plate. (c) LL was measured from the T12 inferior end plate to S1 superior end plate by the Cobb method. (B) Pelvic parameters including pelvic tilt (PT), pelvic incidence (PI), and sacral slope (SS). (a) PT is defined as the angle between a vertical line originating at the center of the bicoxofemoral axis and a line drawn between the same point and the middle of the superior end plate of S1. (b) PI is defined as the angle between the line perpendicular to the sacral plate and the line connecting the midpoint of the sacral plate to the bicoxofemoral axis. (c) SS is the angle between the S1 superior end plate and a horizontal line. 


\section{D gait analysis (dynamic parameters)}

Gait analysis was conducted using a Motion Analysis 3D motion analyzer (Motion Analysis Corp., Santa Rosa, CA, USA). The instrument was calibrated prior to performing the gait analysis on each subject. Markers of diameter 2.5 $\mathrm{cm}$ were attached bilaterally to the bony landmarks of the pelvis and lower extremities, including the L5-S1 intervertebral space, anterior superior iliac spine (ASIS), anterior side of the mid-thigh, mid-point of the lateral knee, anterior side of the mid-tibia, lateral malleolus of the fibula, dorsal side between the second and third metatarsal heads, and the calcaneal area on the same line as the metatarsal marker to assess lower limb kinematics during ambulation. Additionally, markers were attached to the bony landmarks of the $\mathrm{C} 7, \mathrm{~T} 6, \mathrm{~T} 12, \mathrm{~L} 2$, and $\mathrm{L} 5 \mathrm{spi}$ nous processes for assessing kinematics of thoracic and lumbar vertebral segments during ambulation (Fig. 2). The participants went through a dynamic test at a range of self-selected walking speed over a $10-\mathrm{m}$ walkway. The infrared camera determined the location of each marker during ambulation. The real-time data of angular changes was delivered into the SIMM (Software for Interactive Musculoskeletal Modeling) program (Motion Analysis
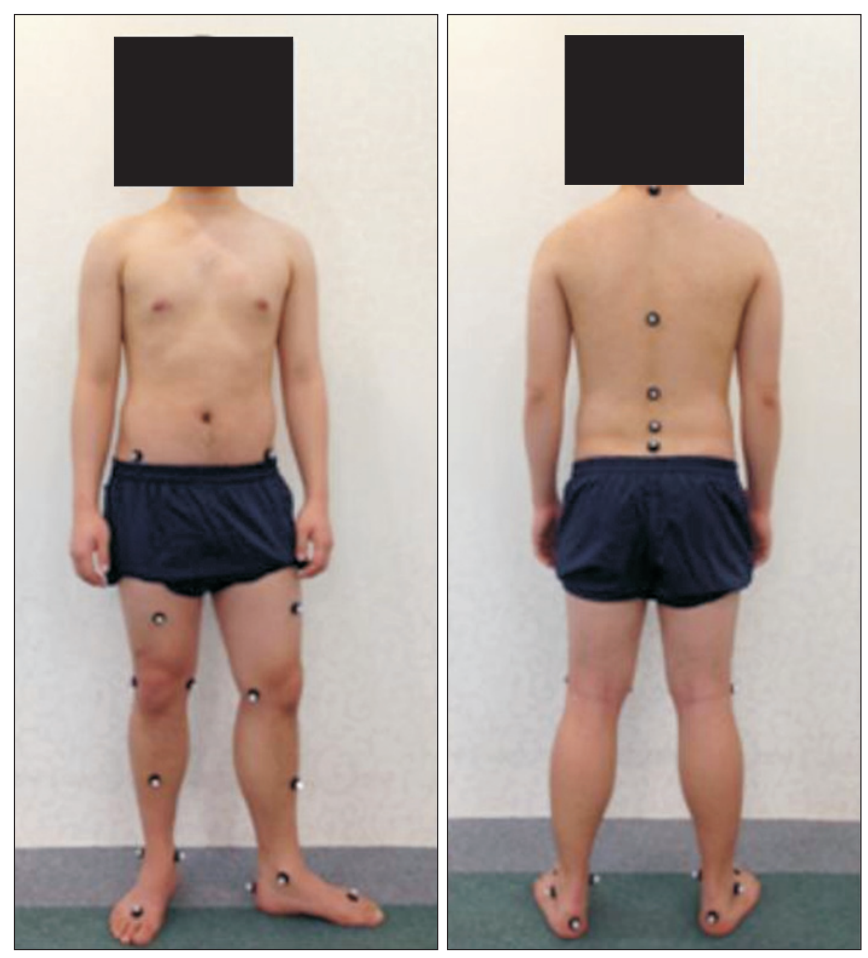

Fig. 2. Location of markers for motion analysis.
Corp.). The angular motions of spinopelvic and lower limb joints, which were continuously changed during ambulation, were analyzed and represented by the SIMM program. Maximal and minimal angles of pelvic tilt, hip, knee and ankle joints in the sagittal plane were measured during ambulation. In addition, maximal and minimal sagittal angles of thoracic vertebral segments (dynamic TK), and lumbar vertebral segments (dynamic LL) were obtained.

\section{Statistical analyses}

Statistical analyses were performed using the SPSS $12.0 \mathrm{~K}$ (SPSS Inc., Chicago, IL, USA). A p-value of less than 0.05 was considered as significant. A chi-square with the Fisher exact test was used to compare the gender ratio between the DFB group and the control group. The static and dynamic parameters of the DFB group were compared with those of the control group using the Student t-test. We also compared the static and dynamic parameters between pre-operation and post-operation with the paired t-test to estimate the degree of improvement of the static and dynamic parameters after corrective surgeries. The correlation between the changes of the static and dynamic parameters after corrective surgeries was determined using the Pearson correlation coefficient. The amount of improvement of the static and dynamic parameters was compared between the successful and unsuccessful surgical outcomes groups using the Student t-test.

\section{RESULTS}

\section{General characteristics of the DFB group}

Forty-seven ( 4 men and 43 women) patients were included in the DFB group. The level of fusion surgeries were at T10-S1 in 3 patients, at T11-S1 in 5, at L1-S1 in 7, at L2-L5 in 1, at L2-S1 in 22, at L2-S2 in 2, and at L3-S1 in 7. There was no significant difference in gender proportion and age between the DFB and the control groups (Table 1).

Comparison of the static and dynamic parameters between the DFB and control groups

The DFB group showed significantly larger LL (larger positive angle means larger kyphotic angle) and smaller TK than the control group. The DFB group had signifi- 
Table 1. Comparison of general characteristics, static parameters, and dynamic parameters between the DFB group and the control group (unit, ${ }^{\circ}$ )

\begin{tabular}{|c|c|c|c|}
\hline & DFB & Control & p-value \\
\hline Gender (male:female) & $4: 43$ & $6: 38$ & 0.514 \\
\hline Age (yr) & $70.15 \pm 5.99$ & $71.05 \pm 6.27$ & 0.487 \\
\hline LL & $8.46 \pm 14.83$ & $-33.81 \pm 11.95$ & $<0.001^{*}$ \\
\hline TLJ & $3.57 \pm 18.05$ & $5.45 \pm 10.91$ & 0.635 \\
\hline TK & $4.72 \pm 10.04$ & $26.95 \pm 10.85$ & $<0.001^{*}$ \\
\hline PI & $52.80 \pm 13.53$ & $53.27 \pm 12.51$ & 0.866 \\
\hline PT & $37.77 \pm 10.09$ & $17.72 \pm 3.11$ & $<0.001^{*}$ \\
\hline SS & $14.81 \pm 11.55$ & $37.89 \pm 11.78$ & $<0.001^{*}$ \\
\hline Max dynamic TK & $15.96 \pm 8.46$ & $27.49 \pm 21.60$ & $<0.001^{*}$ \\
\hline Max dynamic LL & $30.19 \pm 13.51$ & $1.16 \pm 11.08$ & $<0.001^{*}$ \\
\hline Min dynamic TK & $9.55 \pm 7.38$ & $23.86 \pm 14.39$ & $<0.001^{*}$ \\
\hline Min dynamic LL & $22.35 \pm 13.33$ & $1.10 \pm 6.77$ & $<0.001^{*}$ \\
\hline Max pelvis posterior tilt & $20.11 \pm 9.40$ & $9.97 \pm 3.01$ & $<0.001^{*}$ \\
\hline Max Rt hip flexion & $54.46 \pm 11.69$ & $35.65 \pm 5.84$ & $<0.001^{*}$ \\
\hline Max Rt knee flexion & $74.29 \pm 8.65$ & $64.28 \pm 5.30$ & $<0.001^{*}$ \\
\hline Max Rt ankle dorsiflexion & $24.08 \pm 3.88$ & $12.53 \pm 2.69$ & $<0.001^{*}$ \\
\hline Max Lt hip flexion & $54.67 \pm 11.90$ & $36.51 \pm 6.04$ & $<0.001^{*}$ \\
\hline Max Lt knee flexion & $73.32 \pm 7.47$ & $64.6 \pm 5.02$ & $<0.001^{*}$ \\
\hline Max Lt ankle dorsiflexion & $24.84 \pm 3.61$ & $12.99 \pm 3.27$ & $<0.001^{*}$ \\
\hline Min pelvis posterior tilt & $14.33 \pm 9.12$ & $7.53 \pm 2.76$ & $<0.001^{*}$ \\
\hline Min Rt hip flexion & $19.26 \pm 15.00$ & $-8.13 \pm 5.00$ & $<0.001^{*}$ \\
\hline Min Rt knee flexion & $11.88 \pm 6.43$ & $1.38 \pm 4.24$ & $<0.001^{*}$ \\
\hline Min Rt ankle dorsiflexion & $-8.15 \pm 5.70$ & $-16.7 \pm 3.97$ & $<0.001^{*}$ \\
\hline Min Lt hip flexion & $18.56 \pm 14.48$ & $-7.32 \pm 4.02$ & $<0.001^{*}$ \\
\hline Min Lt knee flexion & $12.05 \pm 5.62$ & $1.13 \pm 5.22$ & $<0.001^{*}$ \\
\hline Min Lt ankle dorsiflexion & $-9.29 \pm 5.95$ & $-15.77 \pm 4.52$ & $<0.001^{*}$ \\
\hline
\end{tabular}

DFB, degenerative flat back; LL, lumbar lordosis; TLJ, thoracolumbar junctional lordosis; TK, thoracic kyphosis; PI, pelvic incidence; PT, pelvic tilt; SS, sacral slope; Max, maximal value; Min, minimal value; Rt, right; Lt, left.

${ }^{*} \mathrm{p}<0.05$.

cantly larger PT and smaller SS. The DFB group showed smaller maximal and minimal dynamic TK and larger maximal and minimal dynamic LL than the control group. The DFB group displayed increased maximal and minimal pelvic posterior tilt, hip flexion, knee flexion, and ankle dorsiflexion angles than the control group (Table 1).

Improvement of static and dynamic parameters in the DFB group after fusion surgery

In the DFB group showed that mean LL changed from $8.46^{\circ}$ to $-31.44^{\circ}$ after corrective surgery, which was statistically significant. The mean TK was also significantly changed from $4.72^{\circ}$ to $18.73^{\circ}$ after surgery. The preoperative mean PI and SS were measured as $52.8^{\circ}$ and $14.81^{\circ}$, respectively and were significantly increased into $63.62^{\circ}$ and $31.43^{\circ}$ after surgery. As well, the mean PT was significantly decreased after surgery (from $37.77^{\circ}$ to $31.93^{\circ}$ ).

In terms of the dynamic parameters, maximal and minimal TK were increased, while maximal and minimal LL decreased significantly after corrective surgery. Maximal pelvic posterior tilt, hip flexion, knee flexion, and ankle dorsiflexion angles were decreased significantly after surgery. As well, minimal pelvic posterior tilt, hip flexion, and knee flexion were decreased significantly after surgery (Table 2). 
Correlation of static and dynamic parameters of the DFB group

The change $(\Delta)$ of maximal dynamic TK was significantly correlated with the $\Delta \mathrm{LL}$ and $\Delta \mathrm{TK}$ measured on the simple radiography. The $\Delta$ minimal dynamic TK also showed a significant correlation with the $\Delta \mathrm{LL}, \Delta \mathrm{TLJ}$, and $\Delta \mathrm{TK}$. $\Delta$ Maximal and minimal dynamic LL was correlated with only $\Delta \mathrm{TK}$. $\Delta$ Minimal pelvic posterior tilt was correlated with $\Delta \mathrm{TK}$ (Table 3 ).
Comparison of static and dynamic parameters between successful and unsuccessful surgeries

There were no significant differences in the static and dynamic parameters at pre-operation between the successful and unsuccessful surgical groups. Comparing the amount of the improvement acquired by surgeries between the two groups, the successful surgical group obtained significantly greater improvement of the $\Delta$ maximal and minimal dynamic LL than the unsuccessful surgical group. Otherwise, no significant difference was found in the static parameters between the successful and unsuccessful surgical groups (Table 4).

Table 2. Improvement of static and dynamic parameters of the DFB group between pre-operation and post-operation $\left(\right.$ unit, $^{\circ}$ )

\begin{tabular}{|c|c|c|c|}
\hline & Pre-operation & Post-operation & p-value \\
\hline LL & $8.46 \pm 14.83$ & $-31.44 \pm 17.15$ & $<0.001^{*}$ \\
\hline TLJ & $3.57 \pm 18.05$ & $9.84 \pm 19.51$ & 0.058 \\
\hline TK & $4.72 \pm 10.04$ & $18.73 \pm 14.24$ & $<0.001^{*}$ \\
\hline PI & $52.80 \pm 13.53$ & $63.62 \pm 12.30$ & $<0.001^{*}$ \\
\hline PT & $37.77 \pm 10.09$ & $31.93 \pm 10.49$ & $0.002^{*}$ \\
\hline SS & $14.81 \pm 11.55$ & $31.43 \pm 11.84$ & $<0.001^{*}$ \\
\hline Max dynamic TK & $15.96 \pm 8.46$ & $37.58 \pm 18.18$ & $<0.001^{*}$ \\
\hline Max dynamic LL & $30.19 \pm 13.51$ & $6.23 \pm 8.03$ & $<0.001^{*}$ \\
\hline Min dynamic TK & $9.55 \pm 7.38$ & $29.64 \pm 17.36$ & $<0.001^{*}$ \\
\hline Min dynamic LL & $22.35 \pm 13.33$ & $-0.29 \pm 7.27$ & $<0.001^{*}$ \\
\hline Max pelvis posterior tilt & $20.11 \pm 9.40$ & $10.77 \pm 5.42$ & $<0.001^{*}$ \\
\hline Max Rt hip flexion & $54.46 \pm 11.69$ & $41.1 \pm 22.41$ & $<0.001^{*}$ \\
\hline Max Rt knee flexion & $74.29 \pm 8.65$ & $64.91 \pm 7.34$ & $<0.001^{*}$ \\
\hline Max Rt ankle dorsiflexion & $24.08 \pm 3.88$ & $20.63 \pm 5.45$ & $<0.001^{*}$ \\
\hline Max Lt hip flexion & $54.67 \pm 11.90$ & $40.76 \pm 22.50$ & $<0.001^{*}$ \\
\hline Max Lt knee flexion & $73.32 \pm 7.47$ & $63.60 \pm 7.83$ & $<0.001^{*}$ \\
\hline Max Lt ankle dorsiflexion & $24.84 \pm 3.61$ & $19.87 \pm 4.09$ & $<0.001^{*}$ \\
\hline Min pelvis posterior tilt & $14.33 \pm 9.12$ & $4.04 \pm 5.92$ & $<0.001^{*}$ \\
\hline Min Rt hip flexion & $19.26 \pm 15.00$ & $-9.65 \pm 26.98$ & $<0.001^{*}$ \\
\hline Min Rt knee flexion & $11.88 \pm 6.43$ & $3.79 \pm 8.81$ & $<0.001^{*}$ \\
\hline Min Rt ankle dorsiflexion & $-8.15 \pm 5.70$ & $-9.61 \pm 5.19$ & 0.12 \\
\hline Min Lt hip flexion & $18.56 \pm 14.48$ & $-9.53 \pm 26.84$ & $<0.001^{*}$ \\
\hline Min Lt knee flexion & $12.05 \pm 5.62$ & $3.81 \pm 7.12$ & $<0.001^{*}$ \\
\hline Min Lt ankle dorsiflexion & $-9.29 \pm 5.95$ & $-10.19 \pm 5.91$ & 0.339 \\
\hline
\end{tabular}

DFB, degenerative flat back; LL, lumbar lordosis; TLJ, thoracolumbar junctional lordosis; TK, thoracic kyphosis; PI, pelvic incidence; PT, pelvic tilt; SS, sacral slope; Max, maximal value; Min, minimal value; Rt, right; Lt, left. ${ }^{*} \mathrm{p}<0.05$. 
Table 3. Correlation of change of static and dynamic parameters of the DFB group

\begin{tabular}{|c|c|c|c|c|c|c|c|}
\hline & & $\Delta \mathbf{L L}$ & $\Delta$ TLJ & $\Delta \mathrm{TK}$ & $\Delta \mathbf{P I}$ & $\Delta \mathbf{P T}$ & $\Delta \mathbf{S S}$ \\
\hline \multirow[t]{2}{*}{$\Delta$ Max dynamic TK } & $\mathrm{r}$ & 0.344 & 0.274 & 0.460 & 0.062 & 0.106 & -0.062 \\
\hline & p-value & $0.018^{*}$ & 0.062 & $0.001^{*}$ & 0.678 & 0.479 & 0.680 \\
\hline \multirow[t]{2}{*}{$\Delta$ Min dynamic TK } & $\mathrm{r}$ & -0.376 & -0.300 & 0.515 & -0.066 & -0.125 & 0.071 \\
\hline & p-value & $0.009 *$ & $0.040^{*}$ & $<0.001^{*}$ & 0.659 & 0.402 & 0.637 \\
\hline \multirow[t]{2}{*}{$\Delta$ Max dynamic LL } & $\mathrm{r}$ & 0.133 & -0.062 & -0.395 & 0.058 & 0.045 & 0.020 \\
\hline & p-value & 0.373 & 0.680 & $0.006^{*}$ & 0.698 & 0.764 & 0.895 \\
\hline \multirow[t]{2}{*}{$\Delta$ Min dynamic LL } & $\mathrm{r}$ & 0.086 & -0.117 & -0.398 & -0.017 & -0.027 & 0.018 \\
\hline & $\mathrm{p}$-value & 0.566 & 0.432 & $0.006^{*}$ & 0.912 & 0.856 & 0.904 \\
\hline \multirow[t]{2}{*}{$\Delta$ Max pelvic posterior tilt } & $\mathrm{r}$ & -0.007 & 0.057 & -0.254 & 0.042 & -0.131 & 0.141 \\
\hline & $\mathrm{p}$-value & 0.965 & 0.702 & 0.085 & 0.778 & 0.380 & 0.346 \\
\hline \multirow[t]{2}{*}{$\Delta$ Min pelvic posterior tilt } & $\mathrm{r}$ & 0.072 & 0.049 & -0.316 & -0.102 & -0.100 & 0.025 \\
\hline & $\mathrm{p}$-value & 0.632 & 0.742 & $0.030^{*}$ & 0.496 & 0.504 & 0.865 \\
\hline
\end{tabular}

DFB, degenerative flat back; LL, lumbar lordosis; TLJ, thoracolumbar junctional lordosis; TK, thoracic kyphosis; PI, pelvic incidence; PT, pelvic tilt; SS, sacral slope; Max, maximal value; Min, minimal value; r, correlation coefficient. ${ }^{*} \mathrm{p}<0.05$.

\section{DISCUSSION}

Patients with DFB show sagittal imbalance due to decreased lumbar lordosis and have functional impairments related to daily activities including ambulation. The assessment of the dynamic parameters is more associated with patients' functional aspects. It is assumed that $3 \mathrm{D}$ motion analysis can evaluate spinopelvic and lower limb kinematics in patients with DFB during ambulation and can identify the functional improvement obtained by surgery.

Our study indicated that surgical outcomes were more closely related to be the dynamic parameters, such as dynamic lumbar lordosis. Lumbar lordosis plays an important role in the sagittal alignment and balance so that a successful correction of the lumbar lordosis is critical to the functional improvement or patient satisfaction [8]. Although more significant improvement of the dynamic lumbar lordosis was found in the successful group than in the unsuccessful group, no significant difference was found in the static parameters including lumbar lordosis between the two groups. This result was explained by the fact that the dynamic parameters during ambulation were more related to daily functional activities, and as a result, more influential factor to patient satisfaction. Therefore, the improvement of the dynamic parameters measured by the 3D analysis was more important in predicting the surgical outcomes than angular measure- ments on plain radiography.

Studies have related surgical outcomes to motion analysis. One reported that the posterior pelvic tilt among the dynamic parameters at pre-operation was important in predicting good surgical results after corrective surgeries in the patients with DFB, while none of the radiographic static parameters of the spine indicated any clue to surgical results [5]. Another study reported that the posterior pelvic tilt angle was significantly related to successful outcomes of corrective osteotomy, whereas preoperative static pelvic tilt angle was not [14]. However, these studies did not perform postoperative motion analysis therefore they could not compare the amount of improvement of the dynamic parameters achieved by surgeries between the successful and unsuccessful groups. As well, they did not evaluate the dynamic parameters of the spinal segments.

Compared with normal controls, the DFB group with decreased lumbar lordosis showed lordotic/flat thoracic angle and posteriorly tilted pelvis resulting in reduced SS, which was considered as a compensation mechanism in order to prevent the excessive sagittal malalignment or anterior translation of the body axis $[3,7]$. These compensatory changes could be reversed by corrective surgery of lumbar kyphosis [9]. Our study showed compatible results to these previous literatures and additionally, we identified those compensatory changes at the adjacent segments and reversible corrections after surgery in 
Table 4. Comparison of static and dynamic parameters between successful and unsuccessful surgical group in patients with DFB (unit, ${ }^{\circ}$ )

\begin{tabular}{|lccc}
\hline & Successful $(\mathbf{n}=\mathbf{3 0})$ & Unsuccessful $(\mathbf{n}=\mathbf{1 7})$ & p-value \\
\hline LL & $37.64 \pm 20.20$ & $42.95 \pm 27.23$ & 0.450 \\
\hline TLJ & $7.75 \pm 22.17$ & $4.20 \pm 19.62$ & 0.586 \\
\hline TK & $16.14 \pm 10.84$ & $8.93 \pm 13.52$ & 0.070 \\
\hline PI & $9.21 \pm 12.47$ & $13.64 \pm 15.78$ & 0.294 \\
\hline PT & $6.32 \pm 9.99$ & $5.00 \pm 15.78$ & 0.726 \\
\hline SS & $15.47 \pm 12.95$ & $18.63 \pm 14.39$ & 0.444 \\
\hline Max dynamic TK & $24.69 \pm 17.00$ & $16.40 \pm 11.29$ & 0.081 \\
\hline Max dynamic LL & $27.60 \pm 12.38$ & $17.73 \pm 9.51$ & $0.007^{*}$ \\
\hline Min dynamic TK & $22.61 \pm 16.53$ & $15.79 \pm 11.29$ & 0.139 \\
\hline Min dynamic LL & $26.51 \pm 12.65$ & $16.02 \pm 8.34$ & $0.004^{*}$ \\
\hline Max pelvis posterior tilt & $10.52 \pm 8.68$ & $7.26 \pm 9.59$ & 0.241 \\
\hline Max Rt hip flexion & $13.61 \pm 28.15$ & $12.92 \pm 12.58$ & 0.924 \\
\hline Max Rt knee flexion & $10.63 \pm 8.47$ & $7.17 \pm 8.97$ & 0.194 \\
\hline Max Rt ankle dorsiflexion & $3.97 \pm 5.35$ & $2.53 \pm 7.05$ & 0.436 \\
\hline Max Lt hip flexion & $13.46 \pm 27.33$ & $14.71 \pm 10.71$ & 0.857 \\
\hline Max Lt knee flexion & $10.33 \pm 7.18$ & $8.62 \pm 7.51$ & 0.444 \\
\hline Max Lt ankle dorsiflexion & $5.00 \pm 4.10$ & $4.92 \pm 2.94$ & 0.942 \\
\hline Min pelvis posterior tilt & $11.13 \pm 10.19$ & $8.81 \pm 9.78$ & 0.450 \\
\hline Min Rt hip flexion & $32.63 \pm 34.64$ & $22.35 \pm 13.11$ & 0.247 \\
\hline Min Rt knee flexion & $8.49 \pm 9.05$ & $7.37 \pm 7.33$ & 0.664 \\
\hline Min Rt ankle dorsiflexion & $1.64 \pm 6.55$ & $1.16 \pm 6.14$ & 0.805 \\
\hline Min Lt hip flexion & $32.03 \pm 33.47$ & $21.14 \pm 13.48$ & 0.208 \\
\hline Min Lt knee flexion & $8.08 \pm 6.67$ & $8.51 \pm 6.29$ & 0.829 \\
\hline Min Lt ankle dorsiflexion & $1.14 \pm 6.67$ & $0.49 \pm 6.09$ & 0.742 \\
\hline
\end{tabular}

DFB, degenerative flat back; LL, lumbar lordosis; TLJ, thoracolumbar junctional lordosis; TK, thoracic kyphosis; PI, pelvic incidence; PT, pelvic tilt; SS, sacral slope; Max, maximal value; Min, minimal value; Rt, right; Lt, left. ${ }^{*} \mathrm{p}<0.05$.

terms of not only static parameters but also dynamic parameters (Tables 1, 2).

DFB also leads to the change of the lower limb kinematics in addition to the spinopelvic kinematics. Increased knee flexion and ankle extension (dorsiflexion) associated with DFB have been reported $[7,18,19]$. Our study also revealed more increased hip flexion, knee flexion, and ankle dorsiflexion angles in the DFB group than in the control group. Considering their old age, it was doubtful that the increased lower limb angles of DFB group might be the result of hip and knee osteoarthritis. But, the increased lower limb flexion angles at pre-operation were spontaneously reduced along with the improvement of the spinal angles after lumbar corrective surgeries. Therefore, the larger lower limb flexion angle was a secondary phenomenon resulted from lumbar kyphosis rather than from the joints problems of the lower limbs. It was assumed that the excessive anterior translation of the body center related to the stooped posture in the DFB group would be prevented by such compensatory mechanisms of the lower limb as increased hip and knee flexion, ankle dorsiflexion, which contributed to the posterior pelvic tilt $[6,18]$.

To our knowledge, there has been no published study evaluating the correlation between the changes of improvement of the static and dynamic parameters achieved by surgeries in the patients with DFB. The significant relationship was mainly found between the dynamic and static parameters of the spinal segments. However, no significant relationship was revealed be- 
tween dynamic spine versus static pelvic, dynamic pelvic versus static spine, and dynamic pelvic versus static pelvic parameters. The correlation between the static and dynamic parameters was relatively weak, and therefore, static angular changes measured on X-ray after surgeries had some limitation in predicting the degree of the improvement in the dynamic parameters. Considering that the dynamic parameters were more related to the functional status, the static parameters were not appropriate for the prediction of the functional outcomes after surgeries.

A successful result was defined on the basis of the MacNab criterion of patient subjective satisfaction. Although most of angular deviations of DFB were significantly corrected by corrective fusion surgeries in general, considerable proportion of the patients (36.2\%) was not satisfied with surgical outcomes. There were two possible explanations. First, we established a relatively strict standard in judging successful and unsuccessful group. Only the excellent and good responders were included as the successful group. Second, our study identified that the dynamic lumbar lordosis was the only parameter that was related to the patients' subjective satisfaction among various parameters. Thus, those who did not show enough improvement of the dynamic lumbar lordosis were not satisfied with surgical results, in spite of general improvement of other parameters. This suggested that successful acquisition of enough dynamic lumbar lordosis was the most important factor which determined the success of corrective surgeries in terms of the patients' satisfaction and should be considered as the main goal of corrective surgery.

This study has several limitations. First, we conducted the motion analysis only in terms of ambulation. The tests about other daily activities regarding sit to stand, trunk flexion, or pick up object can provide more useful information related to the patients' functional aspects. Second, the motion analysis was done only within 6 months after the surgery. A 1- or 2-year follow-up study could assess the change of patients' functional status in terms of long-term surgical outcomes.

In conclusion, the patients with DFB showed increased TK and decreased SS in the static parameters and increased posterior pelvic tilt, hip flexion, and knee flexion angles in the dynamic parameters, which could be reversed by lumbar corrective surgeries. The correlation between the static and dynamic parameters was mainly found in the spinal parameters. Surgical outcomes in terms of the patients' satisfaction were more related to the improvement of the dynamic parameters rather than the improvement of the static parameters. Therefore, 3D motion analysis was clinically useful in the evaluation of the patients with DFB in that it allowed for the assessment of the dynamic parameters of the spinopelvic and lower limb segments, which was related to the daily function or treatment outcomes.

\section{CONFLICT OF INTEREST}

No potential conflict of interest relevant to this article was reported.

\section{REFERENCES}

1. Lee JC, Cha JG, Kim Y, Kim YI, Shin BJ. Quantitative analysis of back muscle degeneration in the patients with the degenerative lumbar flat back using a digital image analysis: comparison with the normal controls. Spine (Phila Pa 1976) 2008;33:318-25.

2. Takemitsu Y, Harada Y, Iwahara T, Miyamoto M, Miyatake Y. Lumbar degenerative kyphosis: clinical, radiological and epidemiological studies. Spine (Phila Pa 1976) 1988;13:1317-26.

3. Bae JS, Jang JS, Lee SH, Kim JU. Radiological analysis of lumbar degenerative kyphosis in relation to pelvic incidence. Spine J 2012;12:1045-51.

4. Kang CH, Shin MJ, Kim SM, Lee SH, Lee CS. MRI of paraspinal muscles in lumbar degenerative kyphosis patients and control patients with chronic low back pain. Clin Radiol 2007;62:479-86.

5. Lee CS, Lee CK, Kim YT, Hong YM, Yoo JH. Dynamic sagittal imbalance of the spine in degenerative flat back: significance of pelvic tilt in surgical treatment. Spine (Phila Pa 1976) 2001;26:2029-35.

6. Sarwahi V, Boachie-Adjei O, Backus SI, Taira G. Characterization of gait function in patients with postsurgical sagittal (flatback) deformity: a prospective study of 21 patients. Spine (Phila Pa 1976) 2002;27:2328-37.

7. Barrey C, Roussouly P, Perrin G, Le Huec JC. Sagittal balance disorders in severe degenerative spine. Can we identify the compensatory mechanisms? Eur Spine J 2011;20 Suppl 5:626-33. 
8. Jang JS, Lee SH, Min JH, Maeng DH. Changes in sagittal alignment after restoration of lower lumbar lordosis in patients with degenerative flat back syndrome. J Neurosurg Spine 2007;7:387-92.

9. Jang JS, Lee SH, Min JH, Maeng DH. Influence of lumbar lordosis restoration on thoracic curve and sagittal position in lumbar degenerative kyphosis patients. Spine (Phila Pa 1976) 2009;34:280-4.

10. Khodadadeh S, Eisenstein SM. Gait analysis of patients with low back pain before and after surgery. Spine (Phila Pa 1976) 1993;18:1451-5.

11. McGinley JL, Baker R, Wolfe R, Morris ME. The reliability of three-dimensional kinematic gait measurements: a systematic review. Gait Posture 2009;29:3609.

12. Shum GL, Crosbie J, Lee RY. Three-dimensional kinetics of the lumbar spine and hips in low back pain patients during sit-to-stand and stand-to-sit. Spine (Phila Pa 1976) 2007;32:E211-9.

13. Suda Y, Saitou M, Shibasaki K, Yamazaki N, Chiba K, Toyama Y. Gait analysis of patients with neurogenic intermittent claudication. Spine (Phila Pa 1976) 2002;27:2509-13.

14. Kim WJ, Kang JW, Kang SI, Sung HI, Park KY, Park JG, et al. Factors affecting clinical results after corrective osteotomy for lumbar degenerative kyphosis. Asian Spine J 2010;4:7-14.

15. Macnab I. Negative disc exploration: an analysis of the causes of nerve-root involvement in sixty-eight patients. J Bone Joint Surg Am 1971;53:891-903.

16. Ahn Y, Lee SH, Park WM, Lee HY, Shin SW, Kang HY. Percutaneous endoscopic lumbar discectomy for recurrent disc herniation: surgical technique, outcome, and prognostic factors of 43 consecutive cases. Spine (Phila Pa 1976) 2004;29:E326-32.

17. Legaye J, Duval-Beaupère G, Hecquet J, Marty C. Pelvic incidence: a fundamental pelvic parameter for three-dimensional regulation of spinal sagittal curves. Eur Spine J 1998;7:99-103.

18. Barrey C, Roussouly P, Le Huec JC, D’Acunzi G, Perrin G. Compensatory mechanisms contributing to keep the sagittal balance of the spine. Eur Spine J 2013;22 Suppl 6:S834-41.

19. Obeid I, Hauger O, Aunoble S, Bourghli A, Pellet N, Vital JM. Global analysis of sagittal spinal alignment in major deformities: correlation between lack of lumbar lordosis and flexion of the knee. Eur Spine J 2011;20 Suppl 5:681-5. 